\title{
Accelerator Optimization Using a Network Control and Acquisition System
}

\author{
Cameron G.R. Geddes, Palma E. Catravas, Jerome Faure, Csaba Toth, \\ Jeroen VanTilborg, Wim P. Leemans
}

L'OASIS Group, Lawrence Berkeley National Laboratory, Berkeley CA 94720

\begin{abstract}
Accelerator optimization requires detailed study of many parameters, indicating the need for remote control and automated data acquisition systems. A control and data acquisition system based on a network of commodity PCs and applications with standards based interapplication communication is being built for the l'OASIS accelerator facility. This system allows synchronous acquisistion of data at high $(>1 \mathrm{~Hz})$ rates and remote control of the accelerator at low cost, allowing detailed study of the acceleration process.
\end{abstract}

\section{INTRODUCTION}

Experiments at several facilities have demonstrated that laser driven plasma wakes can provide high acceleration gradients, and can accelerate particles to energies near $100 \mathrm{MeV}$ over $\mathrm{mm}$ scale distances $[1,2,3]$. However, the importance and interactions of major factors in the acceleration process are only beginning to be explored. Contributions of such factors as plasma density and gradients, laser focal location and spotsize, laser chirp and power, as well as various injection schemes need to be evaluated and optimized [3]. In order to do this, experiments at high rep rates are needed in order to scan these and other parameters and to investigate their effects on beam charge, energy, and so on.

In this paper, we describe a control system under development using inexpensive standard components at the l'OASIS facility at Lawrence Berkeley Lab which allows high rep rate experiments in conjunction with a $10 \mathrm{TW} 10 \mathrm{~Hz}$ multi arm laser. Such control systems are increasingly important as experimental laser acceleration progresses from proof of principle to detailed physics and optimization.

\section{SYSTEM DESCRIPTION AND OPERATION}

The l'OASIS laser facility is a multi arm $800 \mathrm{~nm}$ Ti:Saphire laser with compressed beams of 10,3 , and $1 \mathrm{TW}$ at $50 \mathrm{fs}$ with independent compressors, an uncompressed $2 \mathrm{GW}$ beam at $200 \mathrm{ps}$, and a frequency doubled probe beam. All beams operate at $10 \mathrm{~Hz}$ from a single oscillator. The compressed beams are configured to provide a main drive beam for wake generation, an orthogonally polarized coaxial beam for pre ionization or diagnostics, and a counter propagating beam for injection experiments[4]. The uncompressed beam is cylindrically focused to provide heating 


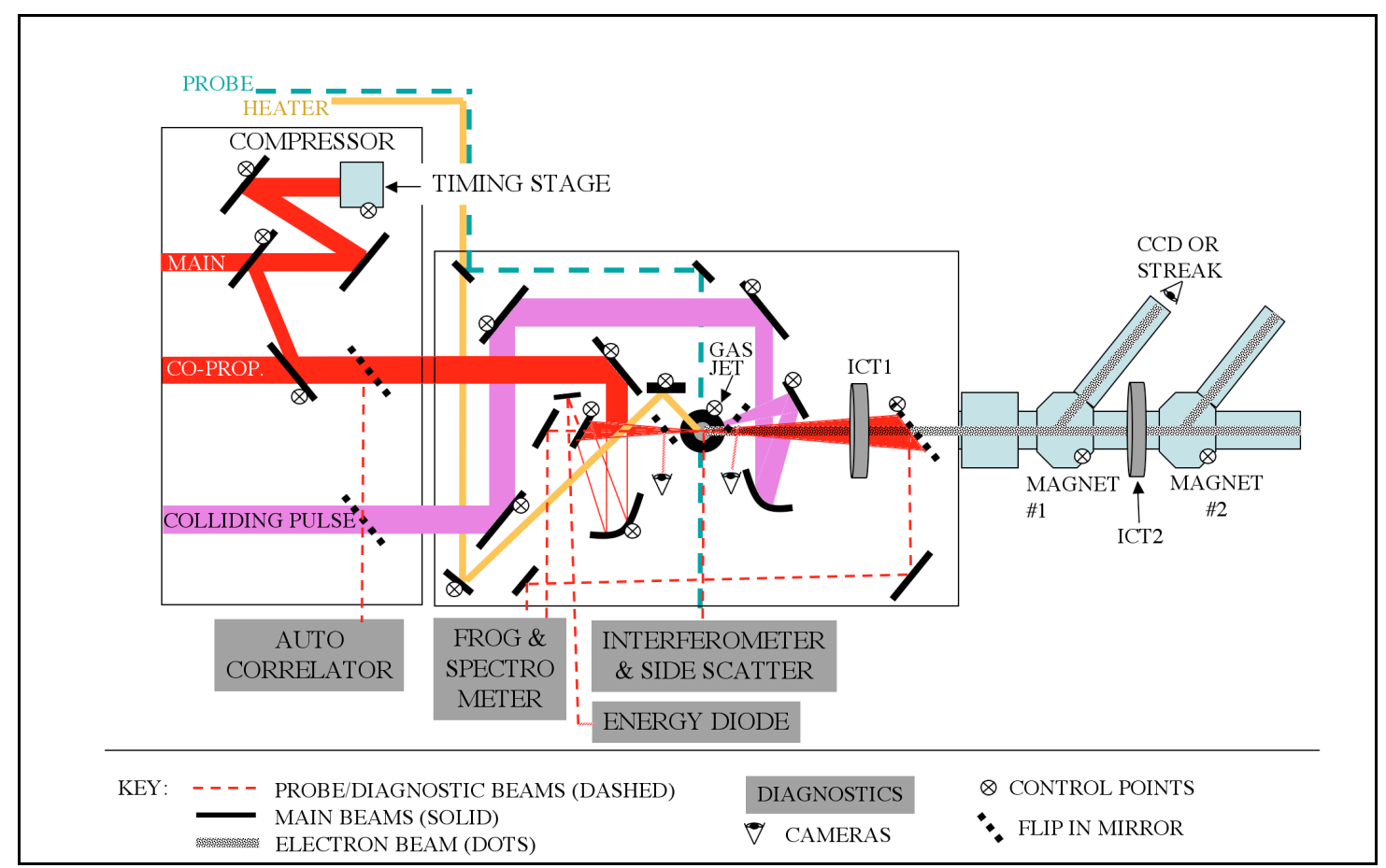

Figure 1. Experimental arrangement of the target chamber (center) and one compressor chamber, showing a selection of major detectors and controlled systems. Radiation detectors are distributed around the area. Nuclear activation targets and THz detectors are not shown.

for channel formation [5], and the frequency doubled beam is used as a probe. This set up is illustrated in figure 1. Because of radiation hazards associated with high rep rate operation, experiments must be operated remotely. Hence each beam must be independently and remotely timed and pointed. In order to take advantage of the high rep rate system, data collection and storage also need to be automated.

The control system developed for this laser is based on a network of personal computers running various custom and commercial control, data collection, and analysis programs, which are coordinated over a 100Base-T Ethernet network. The system comprises a main computer running a control program and several subsidiary machines running various acquisition, control, or analysis software. Ethernet addressable GPIB controllers are used to provide operation of GPIB devices over long distances. Each diagnostic or computer receives a trigger from a timing box timed to assure that they acquire data from the same shot of the laser and gas jet. The control program (written in LabVIEW) running on the main computer remotely coordinates data collection and control on the various machines. By default, each diagnostic is in a 'no acquire' mode. The main control program configures the subsidiary machines according to user instructions, then after each shot it sets a user defined group of the diagnostics to acquire for the next shot over the network. This avoids the need for a hardware triggering system. In fact, the whole system runs on the same hardware required to do individual shot experiments with manual saving of the data, allowing greatly improved data collection efficiency at low cost. Diagnostics capable of keeping pace with the shot rate - currently all but the Photometrics cameras- are acquired every shot. Slow diagnostics are sub cycled; they are fired all at once, and 
fired again only when all of them have finished acquiring to maximize the number of shots on which all diagnostics acquire at once.

The control program uses the DataSocket protocol, a simple Ethernet based messaging system, to communicate with LabVIEW programs running on the subsidiary machines. For diagnostics with open interfaces that allow easy communication, these LabVIEW programs collect data directly. DDE, a Windows inter-process communication tool, is used to communicate with commercial acquisition programs for systems which need specialized software, such as $\mathrm{V}++$ for Photometrics cameras. In this case, the control system uses DataSocket to talk over the network with an intermediary program on the target machine, which in turn controls other acquisition software using DDE. Data acquired is re-distributed by DataSocket and saved to a flat-file database (except for images which are saved directly to a file server), allowing on line plots/analysis from anywhere on the network. Figure 2 shows the system layout and flow of data. Control systems are distributed in the same way. Using higher level languages and protocols such as LabVIEW and DDE, as well as taking advantage of commercial software such as $\mathrm{V}++$ where available, minimized coding time and allowed the system to be put on line by physicists rather than expert developers. This reduced costs and provides flexibility for on going modifications.

A variety of diagnostics and controls are supported. The main control program directly acquires data over GPIB from up to three Tektronix oscilloscopes which read laser energy diodes, beam charge transformers, and other sensors. It also acquires data from position sensors using an A/D board (which also provides triggering) as well as chamber pressure data via a serial interface to a gauge controller. Radiation detectors,

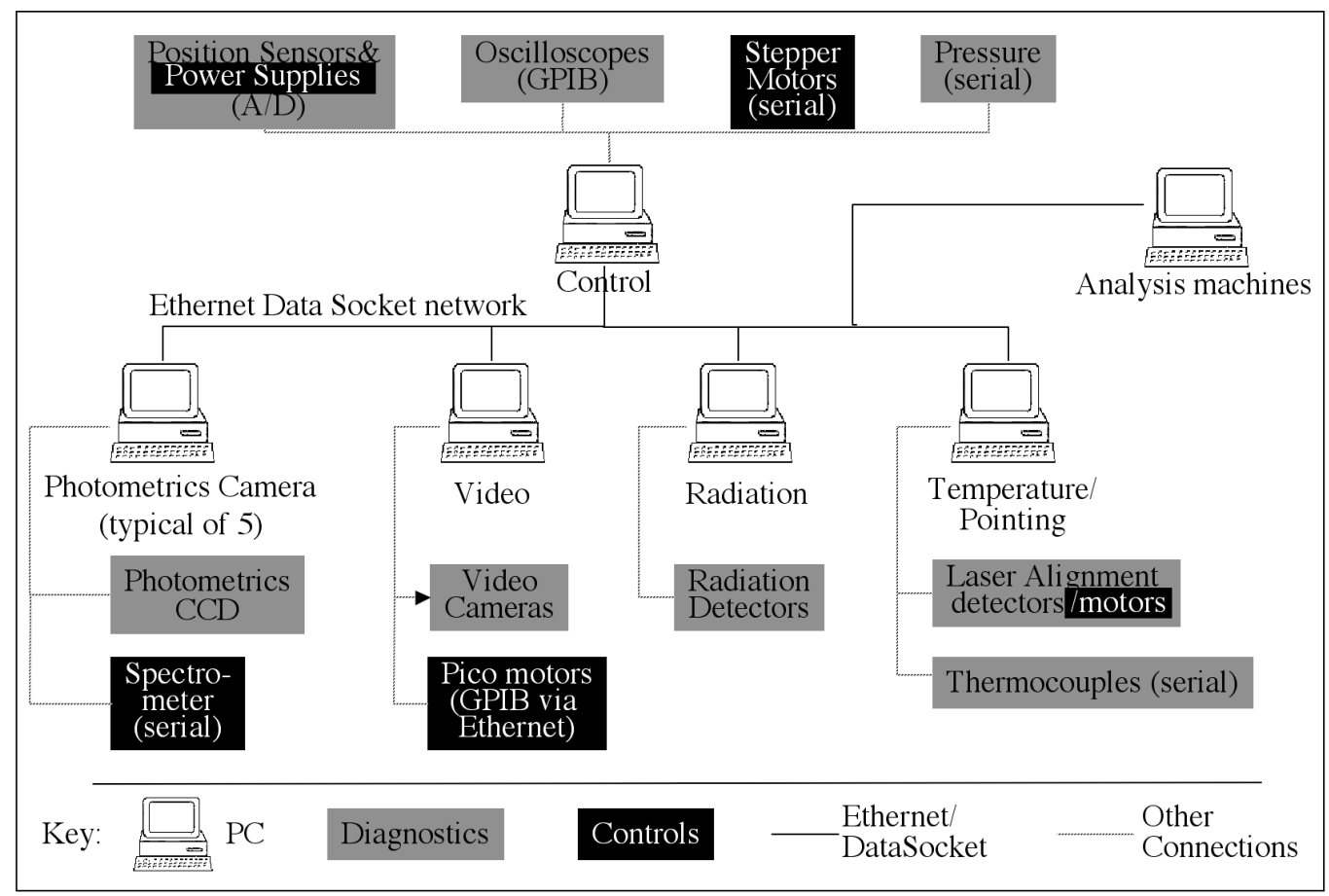

Figure 2. Schematic diagram of the control system, showing representative machines as well as the data and control connections. Not all machines are shown. 
which are used as indicators of high energy electron production, are operated by a second computer. Up to 5 Photometrics digital CCD cameras, each with its own controller computer, are supported via a DataSocket to DDE conversion LabVIEW program which allows the control program to remotely drive the operation of Photometrics' proprietary V++ driver software. Two RS170 analog video framegrabbers reside on another machine. A laser pointing system that uses feedback from quadrature detectors to compensate for beam drift, and a room temperature logging system are also incorporated. A lay out of the main systems in the target area is shown in figure 1 .

The control portion of the system regulates motors and power supplies to allow remote operation of the experiment. Compressor grating position is adjustable to control pulse length and chirp. All beams have independent timing provided by stepper driven stages with $10 \mathrm{fs}$ precision, and automatic compensation to maintain timing during compressor movements is provided by sensing the compressor position. The beams can also be pointed remotely, and the pointing can be monitored via top and side imagers, or via flip in mirrors with microscope objectives. This allows for controlled injection experiments using the colliding pulse concept as well as guiding experiments using plasma channels. The gas jet can be moved in three axes to provide scans of plasma density, scale length, or laser waist location in the profile. Power supplies for magnetic spectrometers are controlled to allow profiling of the beam energy. The system can 'scan' parameters, allowing automated collection of a series of data points at various positions.

System rep rate is limited by diagnostic speed, network set up time required to prepare for a shot, and the time taken to save data across the network. Currently, shot rate is $1-2 \mathrm{~Hz}$, principally limited by a slow A/D board on the control computer which takes approximately $300 \mathrm{~ms}$ to acquire data. The next limit is the time required to set up the Photometrics cameras for each shot using the DataSocket/DDE interface, which is about $100 \mathrm{~ms} /$ camera. The oscilloscopes and serial interfaces are relatively fast, taking $\sim 100 \mathrm{~ms}$ total in parallel with the cameras. The video cameras, on a separate machine, take about 2-300ms to process and save data, which can be improved by using a more efficient $\mathrm{C}$ code interface rather than native LabVIEW code. We anticipate that with a new A/D board and a simple hardware switch on the Photometrics camera triggers to replace the pre shot setup we will be able to acquire at 3-5 Hz, consistent with the limits on our gas jet rep rate set by pumping capacity.

\section{PRELIMINARY EXPERIMENTS}

Preliminary experiments using the control system have begun to map dependence of accelerator performance on laser waist location and gas jet density profile in the self modulated regime. Figure 3 shows bunch charge and the signal on a gamma ray detector placed downstream of the target chamber versus laser waist location for two different gas density profiles. Gamma ray production gives a first order indication of high energy electron production, since higher energy electrons produce higher gamma yield. The two profiles produce nearly identical peak charges of $0.3 \mathrm{nC}$. However, the profile with the steeper gradient and slightly lower peak density (right) produces 

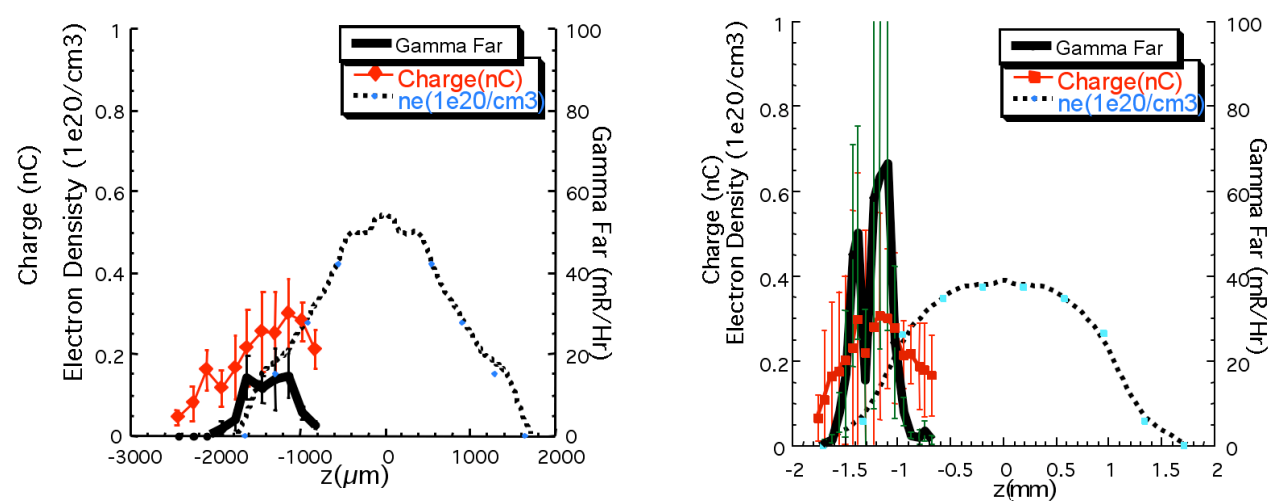

FIGURE 3. Bunch charge and gamma ray yield versus laser waist location shows sensitive dependence on both the waist location and the gas profile. A flat top profile (right) with a steep rise produces nearly the same charge but five times the gamma yield as a more Gaussian profile (left).

approximately five times the gamma yield, indicating production of substantially higher electron energies. There is sensitive dependence for both profiles on the location of the laser waist. The scatter in the data is also different in the two cases, indicating a difference in the stability of the acceleration. Further experiments as well as simulations to understand and optimize the gas profile for best acceleration are under way. The sensitive dependencies found demonstrate the importance of precise experimental control and high rep rate acquisition to allow characterization of the available parameter space.

Other recent experiments using this system have explored the optimization of acceleration by manipulating the laser chirp and laser pulse envelope, as reported by C. Toth in these proceedings.

\section{CONCLUSIONS}

In this paper we have reviewed the control system being developed for the l'OASIS accelerator facility. This control system allows the synchronized, automated collection of data at $\mathrm{Hz}$ rep rates with straightforward extensibility to $\sim 5 \mathrm{~Hz}$. Using this system, detailed studies of accelerator performance are beginning. The detailed studies made possible by the system have already begun to reveal important optimizations that are possible. Future experiments will continue to optimize accelerator performance as well as adding guiding and injection, and will use the detailed data provided by the system to compare to modeling and theoretical predictions.

\section{ACKNOWLEDGMENTS}

This work was supported by the Director, Office of Science, Office of High Energy \& Nuclear Physics, High Energy Physics Division, of the U.S. Department of Energy, under Contract No. DE-AC03-76SF00098. Cameron Geddes is also supported by the 
Hertz foundation. P. Volfbeyn and J. de Groot built original versions of the laser pointing and temperature logging systems incorporated into this system.

\section{REFERENCES}

1. Leemans, W., et. al., submitted to Phys. Rev. Lett.

1. Malka, V., et. al., Phys. Plasmas., 8(6), 2605-08 (2001).

2. Essarey, E., et. al., IEEE Trans. Plasma Sci., PS-24, 252-288 (1996).

3. Volfbeyn, P., et. al., Phys. Plasmas, 6(5), 2269-77 (1999).

4. Esarey, E., et. al., Phys. Rev. Lett.., 79(14), 2682-5 (1997). 